\title{
Spectral analysis for differential systems with a singularity.
}

\section{Mikhail Ignatyev}

Abstract. We consider the differential system $y^{\prime}-x^{-1} A y-q(x) y=\rho B y$ with $n \times n$ matrices $A, B, q(x)$, where $A, B$ are constant, $B$ is diagonal, $A$ and $q(x)$ are off-diagonal, $q(\cdot) \in W_{1}^{1}[0, \infty)$. Some distinguished fundamental system of solutions is constructed. Also, we discuss the inverse scattering problem and obtain the uniqueness result.

Key words: scattering problems, inverse spectral problems, differential systems, singularity AMS Classification: 34A55 34A30 34L25 47E05

1. Introduction. We consider the differential system

$$
y^{\prime}-x^{-1} A y-q(x) y=\rho B y, x>0,
$$

with $n \times n$ matrices $A, B, q(x), x \in(0, \infty)$, where $A, B$ are constant.

Differential equations with coefficients having non-integrable singularities at the end or inside the interval often appear in various areas of natural sciences and engineering. For $n=2$, there exists extensive literature devoted to different aspects of spectral theory of the radial Dirac operators, see, for instance [1], 2], [3], [4], [5].

Systems of the form (1.1) with $n>2$ and arbitrary complex eigenvalues of the matrix $B$ appear to be considerably more difficult for investigation even in the "regular" case $A=0$ [6]. Some difficulties of principal matter also appear due to the presence of singularity at $x=0$. Whereas the "regular" case $A=0$ has been studied fairly completely to date [6], [7], [8], for system (1.1) with $A \neq 0$ there are no similar general results.

In this paper, we concentrate mostly on the construction of a distinguished basis of generalized eigenfunctions for (1.1). We call them the Weyl-type solutions. The Weyl-type solutions play a central role in studying both direct and inverse spectral problems (see, for instance, [9],[10]). In presence of singularity at $x=0$ this step encounters some difficulties that do not appear in a "regular" case $A=0$. In particular, one can not use the auxiliary Cauchy problems with the initial conditions at $x=0$. The approach presented in [11] (see also [12] and references therein) for the scalar differential operators

$$
\ell y=y^{(n)}+\sum_{j=0}^{n-2}\left(\frac{\nu_{j}}{x^{n-j}}+q_{j}(x)\right) y^{(j)}
$$

is based on some special solutions of the equation $\ell y=\lambda y$ that also satisfy certain Volterra integral equations. This approach assumes some additional decay condition for the coefficients $q_{j}(x)$ as $x \rightarrow 0$. In this paper, we do not impose any additional restrictions of such a type. Instead, we use the modification of the approach first presented in [9] for the higher-order differential operators with regular coefficients on the whole line.

In brief outline our approach can be described as follows. We consider some auxiliary systems with respect to the functions with values in the exterior algebra $\wedge \mathbb{C}^{n}$. Our studying of these auxiliary systems centers on two families of their solutions that also satisfy some asymptotical conditions as $x \rightarrow 0$ and $x \rightarrow \infty$ respectively, and can be constructed as solutions of certain Volterra integral equations. As in [9] we call these distinguished tensor solutions the fundamental tensors. The main difference from the above-mentioned method used in [11] is that we use the integral equations to construct the fundamental tensors rather than the solutions for the original system. Since each of the fundamental tensors has minimal 
growth (as $x \rightarrow 0$ or $x \rightarrow \infty$ ) among the solutions of the same auxiliary system, this step does not require any decay of $q(x)$ as $x \rightarrow 0$. As a next step, we show that the fundamental tensors are decomposable. Moreover, they can be represented as the wedge products of some solutions of original system (1.1) and these solutions can be shown to be the Weyl-type solutions of (1.1). This strategy allows us to construct the Weyl-type solutions via purely algebraic procedure and investigate their properties, in particular, their analytical properties with respect to the spectral parameter $\rho$ and asymptotical behavior as $\rho \rightarrow \infty$ and $\rho \rightarrow 0$.

The results obtained can be used in various areas of spectral theory. As an example, we consider the inverse scattering problem for (1.1). For the sake of brevity, we restrict our considerations here only with the uniqueness result, moreover, we assume that the discrete spectrum is empty. A general case and a constructive procedure for solving the inverse problem are planned to be considered in our further works.

Throughout the paper we assume the following

Assumption 1. $B=\operatorname{diag}\left(b_{1}, \ldots, b_{n}\right), n>2, A$ and $q(x)$ are off-diagonal, $q(\cdot) \in$ $W_{1}^{1}[0, \infty)$. The eigenvalues $\left\{\mu_{j}\right\}_{j=1}^{n}$ of matrix $A$ are distinct and such that $\mu_{j}-\mu_{k} \notin \mathbb{Z}$ for $j \neq k$, moreover, $\operatorname{Re} \mu_{1}<\operatorname{Re} \mu_{2}<\ldots<\operatorname{Re} \mu_{n}$. The entries $b_{1}, \ldots, b_{n}$ of matrix $B$ are nonzero distinct complex numbers such that $\sum_{j=1}^{n} b_{j}=0$.

\section{Solutions of the unperturbed system.}

Here we briefly discuss the unperturbed system:

$$
y^{\prime}-x^{-1} A y=\rho B y
$$

and introduce some fundamental systems of its solutions.

We start with considering (2.1) for $\rho=1$

$$
y^{\prime}-x^{-1} A y=B y
$$

but for complex values of $x$.

Let $\Sigma$ be the following union of lines through the origin in $\mathbb{C}$ :

$$
\Sigma=\bigcup_{(k, j): j \neq k}\left\{x: \operatorname{Re}\left(x b_{j}\right)=\operatorname{Re}\left(x b_{k}\right)\right\} .
$$

Then $\mathbb{C} \backslash \Sigma$ can be presented as a union of the sectors $S_{\nu}, \nu=\overline{1, N}$, where $N$ depends on the numbers $b_{1}, \ldots, b_{n}$ and can be from 2 up to $n(n-1)$. For definiteness, we assume that $N>2$.

Consider some (arbitrary) sector $S_{\nu}$.It is well-known that there exists the ordering $R_{1}, \ldots, R_{n}$ of the numbers $b_{1}, \ldots, b_{n}$ such that $\operatorname{Re}\left(R_{1} x\right)<\operatorname{Re}\left(R_{2} x\right) \ldots<\operatorname{Re}\left(R_{n} x\right)$ for any $x \in S_{\nu}$. For $x \in \bar{S}_{\nu} \backslash\{0\}$ we consider the following fundamental matrices for system (2.2):

- $c(x)=\left(c_{1}(x), \ldots, c_{n}(x)\right)$, where

$$
c_{k}(x)=x^{\mu_{k}} \hat{c}_{k}(x),
$$

$\operatorname{det} c(x) \equiv 1$ and all $\hat{c}_{k}(\cdot)$ are entire functions, $\hat{c}_{k}(0)=\mathfrak{h}_{k}, \mathfrak{h}_{k}$ is an eigenvector of the matrix $A$ corresponding to the eigenvalue $\mu_{k}$;

- $e(x)=\left(e_{1}(x), \ldots, e_{n}(x)\right)$, where

$$
e_{k}(x)=\mathrm{e}^{\rho x R_{k}}\left(\mathfrak{f}_{k}+x^{-1} \eta_{k}(x)\right),\left\|\eta_{k}(x)\right\| \leq C_{k},|x| \geq 1, x \in \bar{S}_{\nu},
$$

$\left(\mathfrak{f}_{1}, \ldots, \mathfrak{f}_{n}\right)=\left(\mathfrak{e}_{1}, \ldots, \mathfrak{e}_{n}\right) \Pi_{\nu},\left\{\mathfrak{e}_{1}, \ldots, \mathfrak{e}_{n}\right\}$ is a standard coordinate column basis in $\mathbb{C}^{n}$ and $\Pi_{\nu}$ is a permutation matrix such that $\left(R_{1}, \ldots, R_{n}\right)=\left(b_{1}, \ldots, b_{n}\right) \Pi_{\nu}$. 
Condition $R_{0}$. For all $\nu=\overline{1, N}, k=\overline{1, n}$ the numbers

$$
\Delta_{k}^{0}:=\operatorname{det}\left(e_{1}(x), \ldots, e_{k-1}(x), c_{k}(x), \ldots, c_{n}(x)\right)
$$

are not equal to 0 .

Under Condition $R_{0}$ for $x \in \bar{S}_{\nu} \backslash\{0\}$ there exists (and is unique) the fundamental matrix $\psi^{0}(x)$ such that

$$
\psi_{k}^{0}(x)=\mathrm{e}^{\rho x R_{k}}\left(\mathfrak{f}_{k}+o(1)\right), x \rightarrow \infty, x \in S_{\nu}, \psi_{k}^{0}(x)=O\left(x^{\mu_{k}}\right), x \rightarrow 0
$$

Remark 2.1. The fundamental matrix $c(x)$ is uniquely determined by conditions specified above provided that we fix some set of eigenvectors $\left\{\mathfrak{h}_{k}\right\}_{k=\overline{1, n}}$ of matrix $A$ and some branch for $\arg x$ in $S_{\nu}$. The fundamental matrix $e(\cdot)$ is uniquely determined in any Stokes sector containing $\bar{S}_{\nu} \backslash\{0\}$ but not in $S_{\nu}$ itself. But this does not affect neither Condition $R_{0}$ nor our definition of $\psi^{0}(\cdot)$. Indeed, $e(x)$ for $x \in S_{\nu}$ is determined uniquely up to multiplication by upper-triangular constant matrix with diagonal entries equal to 1 .It is clear that such a multiplication does not change the tensors $e_{1}(x) \wedge \ldots \wedge e_{k-1}(x)$ and therefore the numbers $\Delta_{k}^{0}$. Furthermore, for $\psi^{0}(x)$ one has the representation $\psi^{0}(x)=c(x) l$, where constant lower-triangular matrix $l$ arises from the $L U$-factorization of the matrix $c^{-1}(x) e(x)$. It is clear that multiplication of $e(x)$ by upper-triangular constant matrix with diagonal entries equal to 1 does not change the matrix $l$.

Now we return to (2.1) with arbitrary $\rho \in \bar{S}_{\nu} \backslash\{0\}$ and real positive $x$. Notice that if $y(x)$ satisfies $(2.2)$ then $Y(x, \rho):=y(\rho x)$ satisfies (2.1). Taking this into account, we define the matrix solutions $C(x, \rho), E(x, \rho), \Psi^{0}(x, \rho)$ of $(2.1)$ as follows: $C(x, \rho):=c(\rho x)$, $\Psi^{0}(x, \rho):=\psi^{0}(\rho x), E(x, \rho):=e(\rho x)$.

\section{Fundamental tensors.}

In this section we consider the following auxiliary equations:

$$
Y^{\prime}=Q^{(m)}(x, \rho) Y
$$

where $Y$ is a function with values in the exterior product $\wedge^{m} \mathbb{C}^{n}$. Here and below

$$
Q(x, \rho):=x^{-1} A+\rho B+q(x)
$$

and for given $n \times n$ matrix $M M^{(m)}$ denotes the operator acting in $\wedge^{m} \mathbb{C}^{n}$ so that for any vectors $u_{1}, \ldots, u_{m}$ the following identity holds [9]:

$$
M^{(m)}\left(u_{1} \wedge u_{2} \wedge \ldots \wedge u_{m}\right)=\sum_{j=1}^{m} u_{1} \wedge u_{2} \wedge \ldots \wedge u_{j-1} \wedge M u_{j} \wedge u_{j+1} \wedge \ldots \wedge u_{m} .
$$

In what follows, we shall also use the following notations.

We denote by $\mathcal{A}_{m}$ the set of all ordered multi-indices $\alpha=\left(\alpha_{1}, \ldots, \alpha_{m}\right), \alpha_{1}<\alpha_{2}<\ldots<$ $\alpha_{m}, \alpha_{j} \in\{1,2, \ldots, n\}$. For a set of vectors $u_{1}, \ldots, u_{n}$ from $\mathbb{C}^{n}$ and a multi-index $\alpha \in \mathcal{A}_{m}$ we define

$$
u_{\alpha}:=u_{\alpha_{1}} \wedge \ldots \wedge u_{\alpha_{m}} .
$$

Let $a_{1}, \ldots, a_{n}$ be a numerical sequence. For $\alpha \in \mathcal{A}_{m}$ we define

$$
a_{\alpha}:=\sum_{j \in \alpha} a_{j}
$$


for $k \in \overline{1, n}$ we denote

$$
\vec{a}_{k}:=\sum_{j=1}^{k} a_{j}, \overleftarrow{a}_{k}:=\sum_{j=k}^{n} a_{j}
$$

For a multi-index $\alpha$ the symbol $\alpha^{\prime}$ denotes the ordered multi-index that complements $\alpha$ to $(1,2, \ldots, n)$. We note that Assumption 1 implies, in particular, that $\sum_{k=1}^{n} \mu_{k}=\sum_{k=1}^{n} R_{k}=0$ and therefore for any multi-index $\alpha$ one has $R_{\alpha^{\prime}}=-R_{\alpha}$ and $\mu_{\alpha^{\prime}}=-\mu_{\alpha}$.

It is clear that if a set $\left\{u_{1}, \ldots, u_{n}\right\}$ is a basis in $\mathbb{C}^{n}$ then the set $\left\{u_{\alpha}\right\}_{\alpha \in \mathcal{A}_{m}}$ is a basis in $\wedge^{m} \mathbb{C}^{n}$. In particular the set $\left\{\mathfrak{e}_{\alpha}\right\}_{\alpha \in \mathcal{A}_{m}}$, where $\left\{\mathfrak{e}_{k}\right\}_{k=1}^{n}$ is a standard basis in $\mathbb{C}^{n}$ is a basis in $\wedge^{m} \mathbb{C}^{n}$. In $\wedge^{m} \mathbb{C}^{n}$ we define a norm as follows:

$$
\left\|\sum_{\alpha \in \mathcal{A}_{m}} h_{\alpha} \mathfrak{e}_{\alpha}\right\|:=\sum_{\alpha \in \mathcal{A}_{m}}\left|h_{\alpha}\right| .
$$

Then for $n \times n$ matrix $M$ one has:

$$
\left\|M^{(m)}\right\| \leq m\|M\|
$$

For $h \in \wedge^{n} \mathbb{C}^{n}$ we define $|h|$ as a constant in the following representation:

$$
h=|h| \mathfrak{e}_{1} \wedge \mathfrak{e}_{2} \wedge \ldots \wedge \mathfrak{e}_{n} .
$$

In the sequel we assume that $\rho \in \bar{S}_{\nu}$ for some arbitrary fixed $\nu$. We consider the following Volterra equations:

$$
\begin{gathered}
Y(x)=T_{k}^{0}(x, \rho)+\int_{0}^{x} G_{n-k+1}(x, t, \rho)\left(q^{(n-k+1)}(t) Y(t)\right) d t, \\
Y(x)=F_{k}^{0}(x, \rho)-\int_{x}^{\infty} G_{k}(x, t, \rho)\left(q^{(k)}(t) Y(t)\right) d t
\end{gathered}
$$

where

$$
\begin{gathered}
T_{k}^{0}(x, \rho):=C_{k}(x, \rho) \wedge \ldots \wedge C_{n}(x, \rho), \\
F_{k}^{0}(x, \rho):=E_{1}(x, \rho) \wedge \ldots \wedge E_{k}(x, \rho)=\Psi_{1}^{0}(x, \rho) \wedge \ldots \wedge \Psi_{k}^{0}(x, \rho)
\end{gathered}
$$

and $G_{m}(x, t, \rho)$ is operator acting in $\wedge^{m} \mathbb{C}^{n}$ as follows:

$$
G_{m}(x, t, \rho) f=\sum_{\alpha \in \mathcal{A}_{m}}(-1)^{\sigma_{\alpha}}\left|f \wedge \Psi_{\alpha^{\prime}}^{0}(t, \rho)\right| \Psi_{\alpha}^{0}(x, \rho)=\sum_{\alpha \in \mathcal{A}_{m}}(-1)^{\sigma_{\alpha}}\left|f \wedge E_{\alpha^{\prime}}(t, \rho)\right| E_{\alpha}(x, \rho) .
$$

Here $\sigma_{\alpha} \in\{0,1\}$ is such that $(-1)^{\sigma_{\alpha}}=\left|\mathfrak{f}_{\alpha} \wedge \mathfrak{f}_{\alpha^{\prime}}\right|$.

It follows directly from the definition of the fundamental matrix $\Psi^{0}(x, \rho)$ (see the previous section) that for any $\alpha \in \mathcal{A}_{m}$ the following estimates hold:

$$
\left\|\Psi_{\alpha}^{0}(x, \rho)\right\| \leq C\left\{\begin{array}{l}
\left|(\rho x)^{\mu_{\alpha}}\right|,|\rho x| \leq 1 \\
\left|\exp \left(\rho x R_{\alpha}\right)\right|,|\rho x|>1
\end{array}\right.
$$

Here and below we use the same symbol $C$ to denote different constants that does not depend on $x, \rho$. 
Theorem 3.1. For any $\rho \in \bar{S}_{\nu} \backslash\{0\}$ equations (3.3) and (3.4) have unique solutions $T_{k}(x, \rho)$ and $F_{k}(x, \rho)$ respectively such that

$$
\begin{aligned}
& \left\|T_{k}(x, \rho)\right\| \leq C\left\{\begin{array}{l}
\mid \rho x)^{\overleftarrow{\mu}_{k}}|,| \rho x \mid \leq 1 \\
\exp \left(\rho x \overleftarrow{R}_{k}\right)|,| \rho x \mid>1
\end{array}\right. \\
& \left\|F_{k}(x, \rho)\right\| \leq C\left\{\begin{array}{l}
(\rho x)^{\vec{\mu}_{k}}|,| \rho x \mid \leq 1 \\
\exp \left(\rho x \vec{R}_{k}\right)|.| \rho x \mid>1
\end{array}\right.
\end{aligned}
$$

The following asymptotics hold:

$$
\begin{gathered}
F_{k}(x, \rho)=\exp \left(\rho x \vec{R}_{k}\right)\left(\mathfrak{f}_{1} \wedge \ldots \wedge \mathfrak{f}_{k}+o(1)\right), x \rightarrow \infty \\
T_{k}(x, \rho)=(\rho x)^{\overleftarrow{\mu}_{k}\left(\mathfrak{h}_{k} \wedge \ldots \wedge \mathfrak{h}_{n}+o(1)\right), x \rightarrow 0}
\end{gathered}
$$

Remark 3.1. Notice that $G_{m}(x, t, \rho)$ is the Green's function for the nonhomogeneous equation

$$
Y^{\prime}=Q_{0}^{(m)}(x, \rho) Y+F, Q_{0}(x, \rho)=x^{-1} A+\rho B
$$

i.e., for any $x_{0} \in[0, \infty]$ the integral $Y(x)=\int_{x_{0}}^{x} G_{m}(x, t, \rho) F(t) d t$ (if exists) solves (3.6). Thus the tensors $T_{k}(x, \rho), F_{k}(x, \rho)$ are solutions for (3.1) with $m=n-k+1$ and $m=k$ respectively.

Proof of Theorem 3.1. Let us notice first that $T_{k}^{0}(x, \rho)$ admits the estimates:

$$
\left\|T_{k}^{0}(x, \rho)\right\| \leq C\left\{\begin{array}{l}
(\rho x)^{\overleftarrow{\mu}_{k}}|,| \rho x \mid \leq 1 \\
\exp \left(\rho x \overleftarrow{R}_{k}\right)|,| \rho x \mid>1
\end{array}\right.
$$

Indeed, the first estimate follows directly from the definition of $T_{k}^{0}$, the second one can be obtained from the representation:

$$
T_{k}^{0}(x, \rho)=\sum_{\alpha \in \mathcal{A}_{n-k+1}} T_{k \alpha}^{0} E_{\alpha}(x, \rho)
$$

where the numbers $T_{k \alpha}^{0}$ do not depend on $x, \rho$ and the observation that $\left\|E_{\alpha}(x, \rho)\right\|<$ $C\left|\exp \left(\rho x \overleftarrow{R}_{k}\right)\right|$ for any $\alpha \in \mathcal{A}_{n-k+1},|\rho x|>1$

Now we apply the successive approximation method to (3.3) and consider the sequence $T_{k}^{r}(x, \rho)$ defined in recurrent way as follows:

$$
T_{k}^{r+1}(x, \rho)=\int_{0}^{x} G_{n-k+1}(x, t, \rho)\left(q^{(n-k+1)}(t) T_{k}^{r}(t, \rho)\right) d t .
$$

We use induction in $r$ to obtain the estimate:

$$
\left\|T_{k}^{r}(x, \rho)\right\| \leq C_{0} \cdot \frac{C^{r}}{r !} \cdot\left(\int_{0}^{x}\|q(t)\| d t\right)^{r} \cdot\left\{\begin{array}{l}
(\rho x)^{\overleftarrow{\mu}_{k}}|,| \rho x \mid \leq 1 \\
\exp \left(\rho x \overleftarrow{R}_{k}\right)|,| \rho x \mid>1
\end{array}\right.
$$


The base is obvious since (3.9) for $r=0$ is exactly estimate (3.7). Now let (3.9) be true for some $r$. Then for $|\rho x| \leq 1$ we have:

$$
\left\|T_{k}^{r+1}(x, \rho)\right\| \leq C_{0} \cdot \frac{C^{r}}{r !} \sum_{\alpha \in \mathcal{A}_{n-k+1}} C_{\alpha} \int_{0}^{x}\left|\left(\frac{x}{t}\right)^{\mu_{\alpha}}\right| \cdot\left|(\rho t)^{\overleftarrow{\mu}_{k}}\right| \cdot\|q(t)\| \cdot\left(\int_{0}^{t}\|q(\tau)\| d \tau\right)^{r} d t
$$

Taking into account that for any $\alpha \in \mathcal{A}_{n-k+1}$ one has $\operatorname{Re}\left(\overleftarrow{\mu}_{k}-\mu_{\alpha}\right) \geq 0$ and therefore $\left|(t / x) \overleftarrow{\mu}_{k}-\mu_{\alpha}\right| \leq 1$ for $t \leq x$ we arrive at the first of desired estimates. Further, for $|\rho x|>1$ we write:

$$
\begin{aligned}
& \left\|T_{k}^{r+1}(x, \rho)\right\| \leq C_{0} \cdot \frac{C^{r}}{r !} \sum_{\alpha \in \mathcal{A}_{n-k+1}} C_{\alpha} \int_{0}^{|\rho|^{-1}}\left|\exp \left(\rho x R_{\alpha}\right)\right| \cdot\left|(\rho t)^{\mu_{\alpha^{\prime}}}\right| \cdot\left|(\rho t) \overleftarrow{\mu}_{k}\right| \cdot\|q(t)\| \cdot\left(\int_{0}^{t}\|q(\tau)\| d \tau\right)^{r} d t \\
& +C_{0} \cdot \frac{C^{r}}{r !} \sum_{\alpha \in \mathcal{A}_{n-k+1}} C_{\alpha} \int_{|\rho|^{-1}}^{x}\left|\exp \left(\rho x R_{\alpha}\right)\right| \cdot\left|\exp \left(\rho t R_{\alpha^{\prime}}\right)\right| \cdot\left|\exp \left(\rho t \overleftarrow{R}_{k}\right)\right| \cdot\|q(t)\| \cdot\left(\int_{0}^{t}\|q(\tau)\| d \tau\right)^{r} d t \leq \\
& \leq C_{0} \cdot \frac{C^{r}}{r !} \cdot\left|\exp \left(\rho x \overleftarrow{R}_{k}\right)\right| \sum_{\alpha \in \mathcal{A}_{n-k+1}} C_{\alpha} \int_{0}^{|\rho|^{-1}}\left|\exp \left(\rho x\left(R_{\alpha}-\overleftarrow{R}_{k}\right)\right)\right| \cdot\|q(t)\| \cdot\left(\int_{0}^{t}\|q(\tau)\| d \tau\right)^{r} d t \\
& +C_{0} \cdot \frac{C^{r}}{r !} \cdot\left|\exp \left(\rho x \overleftarrow{R}_{k}\right)\right| \sum_{\alpha \in \mathcal{A}_{n-k+1}} C_{\alpha} \int_{|\rho|^{-1}}^{x}\left|\exp \left(\rho(x-t)\left(R_{\alpha}-\overleftarrow{R}_{k}\right)\right)\right| \cdot\|q(t)\| \cdot\left(\int_{0}^{t}\|q(\tau)\| d \tau\right)^{r} d t
\end{aligned}
$$

where we have taken into account that as above $\left|(\rho t) \overleftarrow{\mu}_{k}-\mu_{\alpha}\right| \leq 1$ if $|\rho t| \leq 1$. Observe that $\operatorname{Re}\left(\rho\left(R_{\alpha}-\overleftarrow{R}_{k}\right)\right) \leq 0$ and therefore in the estimate above one has $\left|\exp \left(\rho x\left(R_{\alpha}-\overleftarrow{R}_{k}\right)\right)\right| \leq 1$ $\left|\exp \left(\rho(x-t)\left(R_{\alpha}-\overleftarrow{R}_{k}\right)\right)\right| \leq 1$. Taking this into account, we obtain the desired estimate for $T_{k}^{r+1}(x, \rho),|\rho x|>1$.

Estimate (3.9) guarantees a convergency of the successive approximation method and yields the desired estimate for $T_{k}(x, \rho)$. Using this estimate and equation (3.3) we obtain specified in theorem asymptotics for $T_{k}(x, \rho)$ as $x \rightarrow 0$.

This completes the proof in what concerns equation (3.3). Equation (3.4) can be considered in a similar way.

In the rest of the section we consider the asymptotical behavior of fundamental tensors $T_{k}(x, \rho), F_{k}(x, \rho)$ for $\rho \rightarrow \infty$ and $\rho \rightarrow 0$.

Lemma 3.1. Let $T \in(0, \infty)$ be arbitrary fixed. Then for the function:

$$
H_{0}(x, \rho):=\int_{0}^{|\rho|^{-1}} G_{n-k+1}(x, t, \rho)\left(q^{(n-k+1)}(t) T_{k}(t, \rho)\right) d t
$$

the following estimate holds:

$$
\left\|H_{0}(x, \rho)\right\| \leq C|\rho|^{-1}\left|\exp \left(\rho x \overleftarrow{R}_{k}\right)\right|
$$

for $|\rho|>T^{-1}$ uniformly in $x \in\left[|\rho|^{-1}, T\right]$, where the constant $C$ depends only on $T$. 
Proof. Using the estimates for $T_{k}(x, \rho)$ from Theorem 3.1 we obtain for $|\rho x| \geq 1$ :

$$
\begin{aligned}
& \left\|H_{0}(x, \rho)\right\| \leq C \sum_{\alpha \in \mathcal{A}_{n-k+1}} \int_{0}^{|\rho|^{-1}}\left|\exp \left(\rho x R_{\alpha}\right)\right| \cdot\left|(\rho t)^{\mu_{\alpha^{\prime}}}\right| \cdot\left|(\rho t)^{\overleftarrow{\mu}_{k}}\right| \cdot\|q(t)\| d t \leq \\
& \leq C\left|\exp \left(\rho x \overleftarrow{R}_{k}\right)\right| \sum_{\alpha \in \mathcal{A}_{n-k+1}} \int_{0}^{|\rho|^{-1}}\left|\exp \left(\rho x\left(R_{\alpha}-\overleftarrow{R}_{k}\right)\right)\right| \cdot\left|(\rho t)^{\overleftarrow{\mu}_{k}-\mu_{\alpha}}\right| \cdot\|q(t)\| d t .
\end{aligned}
$$

As in proof of Theorem 3.1 we notice that $\left|\exp \left(\rho x\left(R_{\alpha}-\overleftarrow{R}_{k}\right)\right)\right| \cdot\left|(\rho t)^{\overleftarrow{\mu}_{k}-\mu_{\alpha}}\right| \leq 1$ and therefore:

$$
\left\|H_{0}(x, \rho)\right\| \leq C \cdot\left|\exp \left(\rho x \overleftarrow{R}_{k}\right)\right| \cdot \max _{x \in\left[0,|\rho|^{-1}\right]}\|q(x)\| \cdot \sum_{\alpha \in \mathcal{A}_{n-k+1}} \int_{0}^{|\rho|^{-1}} d t
$$

for $x \in\left[|\rho|^{-1}, T\right]$.

Lemma 3.2. Let $T \in(0, \infty)$ be arbitrary fixed. Then:

1. for any two multi-indices $\alpha, \beta \in \mathcal{A}_{n-k+1}$ the function:

$$
H_{\alpha \beta}^{0}(x, \rho)=\int_{|\rho|^{-1}}^{x}\left|\left(q^{(n-k+1)}(t) E_{\alpha}(t, \rho)\right) \wedge E_{\beta^{\prime}}(t, \rho)\right| E_{\beta}(x, \rho) d t
$$

admits the estimate:

$$
\left\|H_{\alpha \beta}^{0}(x, \rho)\right\| \leq C\left|\rho^{-\varepsilon} \exp \left(\rho x \overleftarrow{R}_{k}\right)\right|
$$

for $|\rho|>T^{-1}$ uniformly in $x \in\left[|\rho|^{-1}, T\right]$, where $\varepsilon \in(0,1)$ is arbitrary and constant $C$ depends only on $\varepsilon$ and $T$;

2. for any multi-index $\beta \in \mathcal{A}_{k}$ the function:

$$
H_{\beta}^{\infty}(x, \rho)=\int_{|\rho|^{-1}}^{x}\left|\left(q^{(k)}(t) F_{k}^{0}(t, \rho)\right) \wedge E_{\beta^{\prime}}(t, \rho)\right| E_{\beta}(x, \rho) d t
$$

admits the estimate:

$$
\left\|H_{\beta}^{\infty}(x, \rho)\right\| \leq C\left|\rho^{-1} \exp \left(\rho x \vec{R}_{k}\right)\right|
$$

for $|\rho|>T^{-1}$ uniformly in $x \in[T, \infty)$, constant $C$ depends only on $T$.

Proof. For any multi-index $\alpha \in \mathcal{A}_{m} E_{\alpha}(x, \rho)$ admits the representation:

$$
E_{\alpha}(x, \rho)=\exp \left(\rho x R_{\alpha}\right)\left(\mathfrak{f}_{\alpha}+(\rho x)^{-1} \eta_{\alpha}(\rho x)\right),
$$

where $\left\|\eta_{\alpha}(z)\right\| \leq C_{\alpha}$ for any $z \in \bar{S}_{\nu},|z| \geq 1$. Thus we can split $H_{\alpha \beta}^{0}(x, \rho)$ as follows:

$$
H_{\alpha \beta}^{0}(x, \rho)=h_{0}(x, \rho)+h_{1}(x, \rho),
$$




$$
\begin{gathered}
h_{1}(x, \rho)=\int_{|\rho|^{-1}}^{x}(\rho t)^{-1} \exp \left(\rho t R_{\alpha}\right) \cdot\left|\left(q^{(n-k+1)}(t) \eta_{\alpha}(t, \rho)\right) \wedge E_{\beta^{\prime}}(t, \rho)\right| E_{\beta}(x, \rho) d t \\
h_{0}(x, \rho)=h_{00}(x, \rho)+h_{01}(x, \rho), \\
h_{01}(x, \rho)=\int_{|\rho|^{-1}}^{x}(\rho t)^{-1} \exp \left(\rho t R_{\alpha}-\rho t R_{\beta}\right) \cdot\left|\left(q^{(n-k+1)}(t) \mathfrak{f}_{\alpha}\right) \wedge \eta_{\beta^{\prime}}(t, \rho)\right| E_{\beta}(x, \rho) d t \\
h_{001}(x, \rho)=\int_{|\rho|^{-1}}^{x}(\rho x)^{-1} \exp \left(\rho t R_{\alpha}-\rho t R_{\beta}+\rho x R_{\beta}\right) \cdot\left|\left(q^{(n-k+1)}(t) \mathfrak{f}_{\alpha}\right) \wedge \mathfrak{f}_{\beta^{\prime}}\right| \eta_{\beta}(x, \rho) d t \\
h_{000}(x, \rho)=\int_{|\rho|^{-1}}^{x} \exp \left(\rho t R_{\alpha}-\rho t R_{\beta}+\rho x R_{\beta}\right) \cdot\left|\left(q^{(n-k+1)}(t) \mathfrak{f}_{\alpha}\right) \wedge \mathfrak{f}_{\beta^{\prime}}\right| \mathfrak{f}_{\beta} d t .
\end{gathered}
$$

We notice that for $t>|\rho|^{-1}$ and $\varepsilon \in(0,1)$ one has $|\rho t|^{\varepsilon-1}<1$. Thus one can estimate:

$$
\left\|\rho^{\varepsilon} \exp \left(-\rho x \overleftarrow{R}_{k}\right) h_{1}(x, \rho)\right\| \leq C \int_{|\rho|^{-1}}^{x} t^{-\varepsilon}\|q(t)\|\left|\exp \left(\rho t R_{\alpha}-\rho x \overleftarrow{R}_{k}+\rho(x-t) R_{\beta}\right)\right| d t
$$

Let us transform: $t R_{\alpha}-x \overleftarrow{R}_{k}+(x-t) R_{\beta}=(x-t)\left(R_{\beta}-\overleftarrow{R}_{k}\right)+t\left(R_{\alpha}-\overleftarrow{R}_{k}\right)$. Then taking into account that for $\rho \in \bar{S}_{\nu}$ and any $\alpha, \beta \in \mathcal{A}_{n-k+1}$ one has $\operatorname{Re}\left(\rho\left(R_{\alpha}-\overleftarrow{R}_{k}\right)\right) \leq 0, \operatorname{Re}\left(\rho\left(R_{\beta}-\right.\right.$ $\left.\left.\overleftarrow{R}_{k}\right)\right) \leq 0$ we conclude that for $0<t<x$ we have $\left|\exp \left(\rho t R_{\alpha}-\rho x \overleftarrow{R}_{k}+\rho(x-t) R_{\beta}\right)\right| \leq 1$ Thus one gets:

$$
\left\|\rho^{\varepsilon} \exp \left(-\rho x \overleftarrow{R}_{k}\right) h_{1}(x, \rho)\right\| \leq C \max _{t \in[0, T]}\|q(t)\| \cdot \int_{0}^{T} t^{-\varepsilon} d t
$$

The functions $h_{01}(x, \rho)$ and $h_{001}(x, \rho)$ can be estimated in a similar way (in the last case one should notice first that $x^{-1} \leq t^{-1}$ for $\left.t \in\left[|\rho|^{-1}, x\right]\right)$ and we obtain:

$$
\left\|H_{\alpha \beta}^{0}(x, \rho)-h_{000}(x, \rho)\right\| \leq C|\rho|^{-\varepsilon}\left|\exp \left(\rho x \overleftarrow{R}_{k}\right)\right|
$$

Now we consider $h_{000}(x, \rho)$ and we notice first that $h_{000}(x, \rho) \equiv 0$ if $\alpha=\beta$. Indeed, we have:

$$
q^{(n-k+1)}(t) \mathfrak{f}_{\alpha}=\sum_{\gamma \in \mathcal{A}_{n-k+1}} q_{\gamma \alpha}^{(n-k+1)}(t) \mathfrak{f}_{\gamma}
$$

with complex (scalar) coefficients $q_{\gamma \alpha}^{(n-k+1)}(t)$. Therefore we can write:

$$
\left|\left(q^{(n-k+1)}(t) \mathfrak{f}_{\alpha}\right) \wedge \mathfrak{f}_{\beta^{\prime}}\right|=\sum_{\gamma \in \mathcal{A}_{n-k+1}} q_{\gamma \alpha}^{(n-k+1)}(t)\left|\mathfrak{f}_{\gamma} \wedge \mathfrak{f}_{\beta^{\prime}}\right|
$$

If $\alpha=\beta$ (3.11) becomes:

$$
\left|\left(q^{(n-k+1)}(t) \mathfrak{f}_{\alpha}\right) \wedge \mathfrak{f}_{\alpha^{\prime}}\right|=\sum_{\gamma \in \mathcal{A}_{n-k+1}} q_{\gamma \alpha}^{(n-k+1)}(t)\left|\mathfrak{f}_{\gamma} \wedge \mathfrak{f}_{\alpha^{\prime}}\right|=q_{\alpha \alpha}^{(n-k+1)}(t)\left|\mathfrak{f}_{\alpha} \wedge \mathfrak{f}_{\alpha^{\prime}}\right|=0
$$


since $q_{j j}(t) \equiv 0, j=\overline{1, n}$ implies $q_{\alpha \alpha}^{(m)}(t) \equiv 0$ for all $m=\overline{1, n}, \alpha \in \mathcal{A}_{m}$.

Now, suppose $\alpha \neq \beta$. Then (3.11) yields:

$$
\left\|h_{000}(x, \rho)\right\|=\left\|\int_{|\rho|^{-1}}^{x} \exp \left(\rho t R_{\alpha}-\rho t R_{\beta}+\rho x R_{\beta}\right) \cdot q_{\beta \alpha}^{(n-k+1)}(t) \mathfrak{f}_{\beta} d t\right\| .
$$

Using integration by parts we obtain:

$$
\begin{gathered}
\left\|h_{000}(x, \rho)\right\| \leq C|\rho|^{-1}|| q(x) \|\left|\exp \left(\rho x R_{\alpha}\right)\right|+C_{1}|\rho|^{-1}\left|\exp \left(\rho x R_{\beta}\right)\right|+ \\
C_{2}|\rho|^{-1} \int_{|\rho|^{-1}}^{x}\left|\exp \left(\rho t R_{\alpha}-\rho t R_{\beta}+\rho x R_{\beta}\right)\right| \cdot\left|p_{\beta \alpha}^{(n-k+1)}(t)\right| d t
\end{gathered}
$$

where $p_{\beta \alpha}^{(n-k+1)}(t) \in L(0, \infty)$. Therefore we have:

$$
\begin{gathered}
\left\|\rho \exp \left(-\rho x \overleftarrow{R}_{k}\right) h_{000}(x, \rho)\right\| \leq C_{1}\left|\exp \left(\rho x\left(R_{\alpha}-\overleftarrow{R}_{k}\right)\right)\right|+C_{2}\left|\exp \left(\rho x\left(R_{\beta}-\overleftarrow{R}_{k}\right)\right)\right|+ \\
C_{3} \int_{|\rho|^{-1}}^{x}\left|\exp \left(\rho t R_{\alpha}-\rho t R_{\beta}+\rho x R_{\beta}-\rho x \overleftarrow{R_{k}}\right)\right| \cdot\left|p_{\beta \alpha}^{(n-k+1)}(t)\right| d t \leq C
\end{gathered}
$$

for $|\rho|>T^{-1}, x \in\left[|\rho|^{-1}, T\right]$ and $C$ depend only on $T$. Together with (3.10) this completes the proof of the first part of the Lemma.

For the function $H_{\beta}^{\infty}(x, \rho)$ one can use the similar arguments. The only difference is that now we don't need to split $(\rho t)^{-1}$ as $(\rho t)^{-\varepsilon} \cdot(\rho t)^{\varepsilon-1}$. For instance, we can estimate (where $\alpha=(1, \ldots, k))$ :

$$
\begin{gathered}
\left\|h_{1}^{\infty}(x, \rho)\right\|:=\left\|\int_{x}^{\infty}(\rho t)^{-1} \exp \left(\rho t R_{\alpha}\right) \cdot\left|\left(q^{(k)}(t) \eta_{\alpha}(t, \rho)\right) \wedge E_{\beta^{\prime}}(t, \rho)\right| E_{\beta}(x, \rho) d t\right\| \leq \\
\leq C\left|\exp \left(\rho x \vec{R}_{k}\right)\right| \int_{x}^{\infty}|\rho t|^{-1}\|q(t)\|\left|\exp \left(\rho(t-x) \vec{R}_{k}+\rho(x-t) R_{\beta}\right)\right| d t \leq \\
\leq C T^{-1}\left|\rho^{-1} \exp \left(\rho x \vec{R}_{k}\right)\right| \int_{T}^{\infty}\|q(t)\| d t .
\end{gathered}
$$

Lemma 3.3. Let $T \in(0, \infty)$ be arbitrary fixed. Then:

1. the following estimate holds:

$$
\left\|T_{k}(x, \rho)-T_{k}^{0}(x, \rho)\right\|<C\left|\rho^{-\varepsilon} \exp \left(\rho x \overleftarrow{R}_{k}\right)\right|
$$

for $|\rho|>T^{-1}$ uniformly in $x \in\left[|\rho|^{-1}, T\right]$, where $\varepsilon \in(0,1)$ is arbitrary and the constant $C$ depend only on $\varepsilon$ and $T$; 
2. the following estimate holds:

$$
\left\|F_{k}(x, \rho)-F_{k}^{0}(x, \rho)\right\|<C\left|\rho^{-1} \exp \left(\rho x \vec{R}_{k}\right)\right|
$$

for $|\rho|>T^{-1}$ uniformly in $x \in[T, \infty)$; the constant $C$ depend only on $T$.

Proof. Let us fix an arbitrary $T>0$ and rewrite equation (3.3) as follows:

$$
\hat{T}_{k}(x, \rho)=\int_{|\rho|^{-1}}^{x} G_{n-k+1}(x, t, \rho)\left(q^{(n-k+1)}(t) \hat{T}_{k}(t, \rho)\right) d t+\hat{T}_{k}^{0}(x, \rho)
$$

where $\hat{T}_{k}(x, \rho):=T_{k}(x, \rho)-T_{k}^{0}(x, \rho), \rho \in \bar{S}_{\nu}$ is arbitrary such that $|\rho|>T^{-1}, x \in\left[|\rho|^{-1}, T\right]$ and

$$
\begin{gathered}
\hat{T}_{k}^{0}(x, \rho)=H_{0}(x, \rho)+H_{1}(x, \rho), \\
H_{0}(x, \rho)=\int_{0}^{|\rho|^{-1}} G_{n-k+1}(x, t, \rho)\left(q^{(n-k+1)}(t) T_{k}(t, \rho)\right) d t \\
H_{1}(x, \rho)=\int_{|\rho|^{-1}}^{x} G_{n-k+1}(x, t, \rho)\left(q^{(n-k+1)}(t) T_{k}^{0}(t, \rho)\right) d t .
\end{gathered}
$$

We use the representations:

$$
\begin{gathered}
G_{n-k+1}(x, t, \rho) f=\sum_{\beta \in \mathcal{A}_{n-k+1}}(-1)^{\sigma_{\beta}}\left|f \wedge E_{\beta^{\prime}}(t, \rho)\right| \cdot E_{\beta}(x, \rho), \\
T_{k}^{0}(t, \rho)=\sum_{\alpha \in \mathcal{A}_{n-k+1}} T_{k \alpha}^{0} E_{\alpha}(t, \rho),
\end{gathered}
$$

where the numbers $T_{k \alpha}^{0}$ do not depend on $t, \rho$ and obtain:

$$
H_{1}(x, \rho)=\sum_{\alpha, \beta \in \mathcal{A}_{n-k+1}}(-1)^{\chi_{k \alpha \beta}} T_{k \alpha}^{0} H_{\alpha \beta}^{0}(x, \rho), \chi_{k \alpha \beta} \in\{0,1\} .
$$

Lemma 3.2 yields the estimate:

$$
\left\|H_{1}(x, \rho)\right\| \leq C|\rho|^{-\varepsilon}\left|\exp \left(\rho x \overleftarrow{R}_{k}\right)\right|
$$

Together with the estimate for $H_{0}(x, \rho)$ from Lemma 3.1 this yields the estimate:

$$
\left\|\hat{T}_{k}^{0}(x, \rho)\right\| \leq C_{0}|\rho|^{-\varepsilon}\left|\exp \left(\rho x \overleftarrow{R}_{k}\right)\right|
$$

where $C_{0}$ depend only on $T$ and $\varepsilon$ and do not depend on $x, \rho$. Then using the successive approximation method to solve (3.12) and proceeding as in the proof of Theorem 3.1 we obtain the estimate:

$$
\left\|\hat{T}_{k}(x, \rho)\right\| \leq C|\rho|^{-\varepsilon}\left|\exp \left(\rho x \overleftarrow{R}_{k}\right)\right|,|\rho|>T^{-1}, x \in\left[|\rho|^{-1}, T\right]
$$

where $C$ do not depend on $x, \rho$. 
The estimate for $F_{k}(x, \rho)$ can be obtained in a similar way using the second part of Lemma 3.2 and successive approximation method applied directly to equation (3.4). hold:

Corollary 3.1. For any fixed $x \in(0, \infty)$ and $\rho \rightarrow \infty, \rho \in \bar{S}_{\nu}$ the following asymptotics

$$
\begin{gathered}
T_{k}(x, \rho)=T_{k}^{0}(x, \rho)+O\left(\rho^{-\varepsilon} \exp \left(\rho x \overleftarrow{R}_{k}\right)\right), \varepsilon \in(0,1) \\
F_{k}(x, \rho)=F_{k}^{0}(x, \rho)+O\left(\rho^{-1} \exp \left(\rho x \vec{R}_{k}\right)\right)
\end{gathered}
$$

We complete this section with the following result concerning a behavior of the fundamental tensors for $\rho \rightarrow 0$. Consider the tensors $\tilde{F}_{k}(x, \rho):=\rho^{-} \vec{\mu}_{k} F_{k}(x, \rho), \tilde{T}_{k}(x, \rho):=$

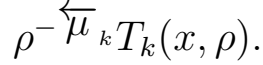

Lemma 3.4. For all $k=\overline{1, n} \tilde{F}_{k}(x, \cdot), \tilde{T}_{k}(x, \cdot)$ admit the continuous extensions to $\bar{S}_{\nu}$.

Proof. First we notice that $\tilde{F}_{k}^{0}(x, \rho):=\rho^{-\vec{\mu}_{k}} F_{k}^{0}(x, \rho), \tilde{T}_{k}^{0}(x, \rho):=\rho^{-\overleftarrow{\mu}_{k}} T_{k}^{0}(x, \rho)$ are continuous w.r.t. $\rho$ in $\bar{S}_{\nu}$. Indeed,

$$
\tilde{T}_{k}^{0}(x, \rho)=\left(x^{\mu_{k}} \hat{c}_{k}(\rho x)\right) \wedge \ldots \wedge\left(x^{\mu_{n}} \hat{c}_{n}(\rho x)\right)
$$

is an entire function of $\rho$. Further, we write $\tilde{F}_{k}(x, \rho)=\tilde{\Psi}_{1}^{0}(x, \rho) \wedge \ldots \wedge \tilde{\Psi}_{k}^{0}(x, \rho)$ (where $\left.\tilde{\Psi}_{k}^{0}(x, \rho):=\rho^{-\mu_{k}} \Psi_{k}^{0}(x, \rho)\right)$ and

$$
\tilde{\Psi}_{j}^{0}(x, \rho)=\sum_{s=j}^{n} l_{s j} \rho^{\mu_{s}-\mu_{j}} x^{\mu_{s}} \hat{c}_{s}(\rho x)
$$

with constant lower-triangular matrix $l$ (see Section 2). Since for $s>j$ one has $\operatorname{Re}\left(\mu_{s}-\mu_{j}\right)>$ 0 , we can conclude that all $\tilde{\Psi}_{k}^{0}(x, \rho)$ and $\tilde{F}_{k}^{0}(x, \rho), k=\overline{1, N}$ are also continuous w.r.t. $\rho \in \bar{S}_{\nu}$.

Now we observe that $\tilde{F}_{k}(x, \cdot), \tilde{T}_{k}(x, \cdot)$ solve the following slightly modified versions of $(3.3),(3.4)$ :

$$
\begin{gathered}
\tilde{T}_{k}(x, \rho)=\tilde{T}_{k}^{0}(x, \rho)+\int_{0}^{x} G_{n-k+1}(x, t, \rho)\left(q^{(n-k+1)}(t) \tilde{T}_{k}(t, \rho)\right) d t \\
\tilde{F}_{k}(x, \rho)=\tilde{F}_{k}^{0}(x, \rho)-\int_{x}^{\infty} G_{k}(x, t, \rho)\left(q^{(k)}(t) \tilde{F}_{k}(t, \rho)\right) d t .
\end{gathered}
$$

Moreover, we can write

$$
G_{m}(x, t, \rho) f=\sum_{\alpha \in \mathcal{A}_{m}}(-1)^{\sigma_{\alpha}}\left|f \wedge \tilde{\Psi}_{\alpha^{\prime}}^{0}(t, \rho)\right| \tilde{\Psi}_{\alpha}^{0}(x, \rho),
$$

and notice that the Green's operator $G_{m}(x, t, \rho)$ is also continuous w.r.t. $\rho \in \bar{S}_{\nu}$. Thus we can repeat the arguments used in the proof of Theorem 3.1, for $\rho \in \bar{S}_{\nu}$.

\section{Weyl-type solutions.}

As in the previous section, we assume $\rho \in S_{\nu}$ for some (arbitrary) $\nu \in \overline{1, N}$.

Definition 4.1. Let $k \in \overline{1, N}$ and $\rho \in S_{\nu}$ be fixed. Function $y(x), x \in(0, \infty)$ is called $k$-th Weyl-type solution if it satisfy (1.1) and the following asymptotics hold:

$$
y(x)=O\left(x^{\mu_{k}}\right), x \rightarrow 0, y(x)=\exp \left(\rho R_{k} x\right)\left(\mathfrak{f}_{k}+o(1)\right), x \rightarrow \infty .
$$


Below in this section we show that the fundamental tensors $F_{k}(x, \rho)$ constructed in previous section can actually be represented as the wedge products of Weyl-type solutions. Our first step is to show that $F_{k}(x, \rho)$ and $T_{k}(x, \rho)$ are decomposable.

Lemma 4.1. For any $\rho \in \bar{S}_{\nu} \backslash\{0\}$ there exist unique sets of absolutely continuous w.r.t. $x \in(0, \infty)$ functions $\left\{v_{1}(x, \rho), \ldots, v_{n}(x, \rho)\right\},\left\{w_{1}(x, \rho), \ldots, w_{n}(x, \rho)\right\}$ such that:

- $\left\{v_{1}, \ldots, v_{n}\right\}$ are orthogonal and $\left\{w_{1}, \ldots, w_{n}\right\}$ are orthogonal;

- $T_{k}=w_{k} \wedge \ldots \wedge w_{n}, F_{k}=v_{1} \wedge \ldots \wedge v_{k}$

- the following asymptotics hold:

$$
v_{k}=\exp \left(\rho R_{k} x\right)\left(\mathfrak{f}_{k}+o(1)\right), x \rightarrow \infty, w_{k}=(\rho x)^{\mu_{k}}\left(\mathfrak{g}_{k}+o(1)\right), x \rightarrow 0,
$$

where $\mathfrak{g}_{n}=\mathfrak{h}_{n}, \mathfrak{g}_{k}-\mathfrak{h}_{k} \in \operatorname{span}\left\{\mathfrak{g}_{j}\right\}_{j>k}$, and vectors $\left\{\mathfrak{g}_{k}\right\}_{k=1}^{n}$ are orthogonal;

- the following relations hold:

$$
\left(w_{k}^{\prime}-Q(x, \rho) w_{k}\right) \wedge T_{k+1}=0, F_{k-1} \wedge\left(v_{k}^{\prime}-Q(x, \rho) v_{k}\right)=0
$$

- $v_{s} \wedge F_{k}=0, w_{k} \wedge T_{s}=0$ if $s \leq k$.

Proof. Assertion of the lemma is similar to Lemma 7.2 [9] and our proof is based in general on the similar ideas. We consider in more detail construction of $\left\{w_{1}, \ldots, w_{n}\right\}$, $\left\{v_{1}, \ldots, v_{n}\right\}$ can be constructed in the same way.

We use an induction in $k=n, n-1, \ldots, 1$. The first step is obvious, since we set $w_{n}:=T_{n}$. Now suppose we have already constructed the vectors $\left\{w_{k+1}, \ldots, w_{n}\right\}$ having the properties specified in the lemma and such that for $j>k T_{j}=w_{j} \wedge \ldots \wedge w_{n}$. Then we apply Lemma 7.1 [9] which asserts that $w_{j} \wedge T_{k}=0$ is a sufficient condition for solvability of the system $w \wedge T_{k+1}=T_{k}$, while the additional requirement $\left(w, w_{j}\right)=0, j=\overline{k+1, n}$ makes the solution unique. Let us denote it as $w_{k}$.

By differentiating the relation $w_{k} \wedge T_{k+1}=T_{k}$ and taking into account that $T_{m}$ satisfies the equation $T_{m}^{\prime}=Q^{(n+1-m)} T_{m}$ we obtain the relation $\left(w_{k}^{\prime}-Q(x, \rho) w_{k}\right) \wedge T_{k+1}=0$.

In order to evaluate an asymptotical behavior of $w_{k}(x, \rho)$ as $x \rightarrow 0$ (while $\rho \in \bar{S}_{\nu} \backslash\{0\}$ is arbitrarily fixed) we use the following representation:

$$
w_{k}(x, \rho)=\sum_{j>k} \beta_{j}(x, \rho) w_{j}(x, \rho)+\sum_{j \leq k} \gamma_{j}(x, \rho) C_{j}(x, \rho) .
$$

Then the relation $w_{k} \wedge T_{k+1}=T_{k}$ becomes:

$$
\sum_{j \leq k} \gamma_{j} C_{j} \wedge T_{k+1}=T_{k}
$$

that yields:

$$
\begin{gathered}
\gamma_{j}\left|C_{1} \wedge \ldots \wedge C_{k} \wedge T_{k+1}\right|= \\
(-1)^{\chi_{j}}\left|C_{1} \wedge \ldots \wedge C_{j-1} \wedge C_{j+1} \wedge \ldots \wedge C_{k} \wedge T_{k}\right|, \chi_{j} \in\{0,1\}, \chi_{k}=0 .
\end{gathered}
$$


Using the asymptotics of $C_{j}$ and $T_{j}$ as $x \rightarrow 0$ we obtain from (4.2):

$$
\gamma_{k}=1+o(1), \gamma_{j}=o\left(\left|(\rho x)^{\mu_{k}-\mu_{j}}\right|\right), j<k
$$

and therefore:

$$
w_{k}^{1}(x, \rho):=\sum_{j \leq k} \gamma_{j} C_{j}=C_{k}(x, \rho)+o\left(\left|(\rho x)^{\mu_{k}}\right|\right)=(\rho x)^{\mu_{k}}\left(\mathfrak{h}_{k}+o(1)\right) .
$$

Now we consider the relation $\left(w_{k}, w_{j}\right)=0, j=\overline{k+1, n}$ that can be written as follows:

$$
\beta_{j}\left(w_{j}, w_{j}\right)+\left(w_{k}^{1}, w_{j}\right)=0 .
$$

In view of (4.3) and the induction assumption this yields:

$$
\beta_{j}(x, \rho)=\left(\beta_{j}^{0}+o(1)\right)(\rho x)^{\mu_{k}-\mu_{j}}, \beta_{j}^{0}\left(\mathfrak{g}_{j}, \mathfrak{g}_{j}\right)+\left(\mathfrak{h}_{k}, \mathfrak{g}_{j}\right)=0 .
$$

Substituting the obtained representations (4.3), (4.4) into (4.1) we obtain:

$$
w_{k}(x, \rho)=(\rho x)^{\mu_{k}}\left(\mathfrak{g}_{k}+o(1)\right), \mathfrak{g}_{k}=\mathfrak{h}_{k}+\sum_{j>k} \beta_{j}^{0} \mathfrak{g}_{j} .
$$

Finally, for any $j>k$ one has: $\left(\mathfrak{g}_{k}, \mathfrak{g}_{j}\right)=\left(\mathfrak{h}_{k}, \mathfrak{g}_{j}\right)+\beta_{j}^{0}\left(\mathfrak{g}_{j}, \mathfrak{g}_{j}\right)=0$ by virtue of $(4.4)$.

In order to complete the induction step we should proof that $w_{k} \wedge T_{j} \equiv 0$ for any $j \leq k$. Denote $w_{k} \wedge T_{j} \equiv 0=: y$, we find that $y(x)$ solves the following equation:

$$
y^{\prime}=Q_{0}^{(n-j+2)}(x, \rho) y+q^{(n-j+2)}(x) y
$$

and admits the estimate:

$$
\|y(x)\| \leq C\left|(\rho x)^{\overleftarrow{\mu_{j}}+\mu_{k}}\right|,|\rho x| \leq 1
$$

This yields the representation:

$$
y(x)=\int_{x_{0}}^{x} G_{n-j+2}(x, t, \rho)\left(q^{(n-j+2)}(t) y(t)\right) d t+\sum_{\alpha \in \mathcal{A}_{n-j+2}} A_{\alpha} \Psi_{\alpha}^{0}(x, \rho),
$$

that is true for any $x_{0}>0$ provided the constants $A_{\alpha}$ are such that:

$$
\sum_{\alpha \in \mathcal{A}_{n-j+2}} A_{\alpha} \Psi_{\alpha}^{0}\left(x_{0}, \rho\right)=y\left(x_{0}\right)
$$

From (4.8) one gets:

$$
\left|A_{\alpha}\right|=\left\|y\left(x_{0}\right) \wedge \Psi_{\alpha^{\prime}}^{0}\left(x_{0}, \rho\right)\right\| .
$$

In view of estimates obtained above for $w_{k}$ and $T_{j}$ this yields for $\left|\rho x_{0}\right|<1$ :

$$
\left|A_{\alpha}\right| \leq C\left|\left(\rho x_{0}\right)^{\overleftarrow{\mu_{j}}+\mu_{k}-\mu_{\alpha}}\right|
$$

Taking the limit as $x_{0} \rightarrow 0$ and taking into account that for any $\alpha \in \mathcal{A}_{n-j+2}$ one has $\operatorname{Re}\left(\overleftarrow{\mu_{j}}+\mu_{k}-\mu_{\alpha}\right)=\operatorname{Re}\left(\overleftarrow{\mu}_{j-1}-\mu_{\alpha}+\mu_{k}-\mu_{j-1}\right) \geq \operatorname{Re}\left(\mu_{k}-\mu_{j-1}\right)>0$ we find that all $A_{\alpha} \rightarrow 0$. Thus we arrive at the following homogeneous Volterra equation with respect to $y$ :

$$
y(x)=\int_{0}^{x} G_{n-j+2}(x, t, \rho)\left(q^{(n-j+2)}(t) y(t)\right) d t .
$$


From (4.9) and estimate (4.6) we obtain successively for $m=1,2, \ldots$ :

$$
\|y(x)\| \leq \frac{C}{m !} \cdot\left|(\rho x)^{\overleftarrow{\mu_{j}}+\mu_{k}}\right| \cdot\left(\int_{0}^{x}\|q(t)\| d t\right)^{m},|\rho x| \leq 1
$$

This means that $y(x)=0$ at least for $x \in\left(0,\left|\rho^{-1}\right|\right)$. Taking into account that $y(x)$ solves a homogeneous first order system of ODE, we conclude that $y(x) \equiv 0$.

Decomposability of the fundamental tensors established above is an important fact for our further considerations. But this is only a preliminary step, since the functions $\left\{v_{k}\right\},\left\{w_{k}\right\}$ do not satisfy (1.1). Moreover, one can notice that they are not holomorphic with respect to $\rho$. Our next (and key) step consists in passing from $\left\{v_{k}\right\}$ to the Weyl-type solutions.

Let us define $\Delta_{k}(\rho)=\left|F_{k-1}(x, \rho) \wedge T_{k}(x, \rho)\right|$. Clear that $\Delta_{k}$ do depend on $x$.

Theorem 4.1. For any $\rho \in \bar{S}_{\nu} \backslash\{0\}$ such that $\Delta_{k}(\rho) \neq 0$ there exists a unique function $\psi_{k}(x, \rho)$ such that:

- $F_{k-1} \wedge \psi_{k}=F_{k}, \psi_{k} \wedge T_{k}=0$

- $\psi_{k}^{\prime}=Q(x, \rho) \psi_{k}$ (i.e. $\psi_{k}$ solves system $\left.(1.1)\right)$

- if $\rho \in S_{\nu}$ then the following asymptotics hold:

$$
\psi_{k}(x, \rho)=\exp \left(\rho R_{k} x\right)\left(\mathfrak{f}_{k}+o(1)\right), x \rightarrow \infty, \psi_{k}(x, \rho)=O\left((\rho x)^{\mu_{k}}\right), x \rightarrow 0 .
$$

Proof. In view of Lemma 4.1 the relations $F_{k-1} \wedge \psi_{k}=F_{k}, \psi_{k} \wedge T_{k}=0$ are equivalent to:

$$
\psi_{k}-v_{k}=\sum_{j<k} \beta_{j} v_{j}, \psi_{k}=\sum_{j \geq k} \gamma_{j} w_{j}
$$

that is actually some system of linear algebraic equations w.r.t. $\left\{\beta_{j}\right\}_{j<k},\left\{\gamma_{j}\right\}_{j \geq k}$ while condition $F_{k-1} \wedge T_{k} \neq 0$ is actually the condition of its unique solvability. Thus, the condition $\Delta_{k}(\rho) \neq 0$ guarantees the existence and uniqueness of $\psi_{k}(x, \rho)$ such that the relations $F_{k-1} \wedge$ $\psi_{k}=F_{k}, \psi_{k} \wedge T_{k}=0$ hold.

To evaluate the asymptotics of $\psi_{k}(x, \rho)$ as $x \rightarrow 0$ we use the representation:

$$
\psi_{k}(x, \rho)=\sum_{j \geq k} \gamma_{j}(x, \rho) w_{j}(x, \rho)
$$

The relation $F_{k-1} \wedge \psi_{k}=F_{k}$ becomes:

$$
\sum_{j \geq k} \gamma_{j} F_{k-1} \wedge w_{j}=F_{k}
$$

that yields:

$$
\gamma_{j} \Delta_{k}=(-1)^{\chi_{j}}\left|F_{k} \wedge w_{k} \wedge \ldots w_{j-1} \wedge w_{j+1} \wedge \ldots \wedge w_{n}\right|, \chi_{j} \in\{0,1\}
$$

Using the estimates for $F_{k}$ from Theorem 3.1 and asymptotics for $w_{j}$ from Lemma 4.1 we obtain $\gamma_{j}=O\left((\rho x)^{\mu_{k}-\mu_{j}}\right)$ for $|\rho x| \leq 1$ and therefore $\psi_{k}(x, \rho)=O\left((\rho x)^{\mu_{k}}\right)$ as $x \rightarrow 0$. 
Now we consider $\psi_{k}(x, \rho)$ for $x \rightarrow \infty$. Here we use the representation:

$$
\psi_{k}(x, \rho)=\sum_{j=1}^{k-1} \beta_{j}(x, \rho) v_{j}(x, \rho)+v_{k}(x, \rho) .
$$

For the coefficients $\beta_{j}$ we have the following equation:

$$
\sum_{j=1}^{k-1} \beta_{j} v_{j} \wedge T_{k}=0
$$

As above, we obtain:

$$
\beta_{j} \Delta_{k}=(-1)^{\chi_{j}}\left|v_{1} \wedge \ldots v_{j-1} \wedge v_{j+1} \wedge \ldots \wedge v_{k-1} \wedge v_{k} \wedge T_{k}\right|, \chi_{j} \in\{0,1\} .
$$

Using the asymptotics of $v_{1}, v_{2}, \ldots$ from Lemma 4.1 and the estimate for $T_{k}$ from Theorem 3.1 one gets:

$$
\begin{gathered}
\beta_{j} \Delta_{k}=(-1)^{\chi_{j}} \delta_{j}+o\left(\exp \left(\rho x\left(R_{k}-R_{j}\right)\right)\right), \delta_{j}:=\left|F_{k, j}^{0} \wedge T_{k}\right|, \\
F_{k, j}^{0}:=E_{1} \wedge \ldots E_{j-1} \wedge E_{j+1} \wedge \ldots \wedge E_{k-1} \wedge E_{k}
\end{gathered}
$$

while for $\delta_{j}$ one has the representation $\delta_{j}=\delta_{j}^{0}+\hat{\delta}_{j}$, where:

$$
\delta_{j}^{0}=\left|F_{k, j}^{0} \wedge T_{k}^{0}\right|
$$

does not depend on $x, \rho$ and

$$
\hat{\delta}_{j}(x, \rho)=\left|F_{k, j}^{0} \wedge \hat{T}_{k}\right| .
$$

Equation (3.3) yields the following representation for $\hat{\delta}_{j}$ :

$$
\hat{\delta}_{j}=\int_{0}^{x}\left|F_{k, j}^{0}(x, \rho) \wedge\left(G_{n-k+1}(x, t, \rho)\left(q^{(n-k+1)}(t) T_{k}(t, \rho)\right)\right) d t\right| .
$$

Now, let us notice that for any $\alpha \in \mathcal{A}_{n-k+1}$ and any system $y_{1}, \ldots, y_{n}$ of solutions of unperturbed equation (2.1) the Wronskian $\left|y_{\alpha} \wedge F_{k, j}^{0}\right|$ does not depend on $x$. Thus, from the representation of the Green's operator $G_{m}(x, t, \rho)$ it follows that:

$$
\hat{\delta}_{j}(x, \rho)=\hat{\delta}_{j 0}(\rho)+\hat{\delta}_{j 1}(x, \rho),
$$

where:

$$
\hat{\delta}_{j 0}(\rho)=(-1)^{\chi_{\alpha}} \int_{0}^{\left|\rho^{-1}\right|}\left|\left(q^{(n-k+1)}(t) T_{k}(t, \rho)\right) \wedge E_{\alpha^{\prime}}(t, \rho)\right| \cdot\left|E_{\alpha}(x, \rho) \wedge F_{k, j}^{0}(x, \rho)\right| d t, \chi_{\alpha} \in\{0,1\},
$$

does not depend on $x$ and:

$$
\hat{\delta}_{j 1}(x, \rho)=(-1)^{\chi_{\alpha}} \int_{\left|\rho^{-1}\right|}^{x}\left|\left(q^{(n-k+1)}(t) T_{k}(t, \rho)\right) \wedge E_{\alpha^{\prime}}(t, \rho)\right| \cdot\left|E_{\alpha}(x, \rho) \wedge F_{k, j}^{0}(x, \rho)\right| d t, \chi_{\alpha} \in\{0,1\} .
$$


Here $\alpha=(j, k+1, \ldots, n)$. Since $\left\|E_{\alpha}(x, \rho) \wedge F_{k, j}^{0}(x, \rho)\right\|=1$, we obtain finally:

$$
\left|\hat{\delta}_{j 1}(x, \rho)\right|=\int_{|\rho|^{-1}}^{x}\left\|\left(q^{(n-k+1)}(t) T_{k}(t, \rho)\right) \wedge E_{\alpha^{\prime}}(t, \rho)\right\| d t .
$$

Using the estimates for $T_{k}$ and $E_{\alpha^{\prime}}$ we obtain:

$$
\left|\hat{\delta}_{j 1}(x, \rho)\right| \leq C \int_{|\rho|^{-1}}^{x}\|q(t)\| \cdot\left|\exp \left(\rho t \overleftarrow{R}_{k}-\rho t R_{\alpha}\right)\right| d t
$$

Since $\overleftarrow{R}_{k}-R_{\alpha}=R_{k}-R_{j}$, we can write:

$$
\left|\hat{\delta}_{j 1}(x, \rho) \exp \left(\rho x\left(R_{j}-R_{k}\right)\right)\right| \leq C \int_{|\rho|^{-1}}^{x}\|q(t)\| \cdot \mid \exp \left(\rho(x-t)\left(R_{j}-R_{k}\right) \mid d t .\right.
$$

For any fixed $\rho \in S_{n}$ and $j<k$ we have $\operatorname{Re} \rho R_{j}<\operatorname{Re} \rho R_{k}$, therefore the right-hand side of the last estimate tends to 0 as $x \rightarrow \infty$. Thus we obtain $\hat{\delta}_{j 1}=o\left(\exp \left(\rho x\left(R_{k}-R_{j}\right)\right)\right)$ and $\hat{\delta}_{j}=o\left(\exp \left(\rho x\left(R_{k}-R_{j}\right)\right)\right)$. This yields $\beta_{j}=\beta_{j}^{0}+o\left(\exp \left(\rho x\left(R_{k}-R_{j}\right)\right)\right)$ and taking again into account that $\operatorname{Re} \rho R_{j}<\operatorname{Re} \rho R_{k}$ we obtain $\beta_{j} v_{j}=o\left(\exp \left(\rho x R_{k}\right)\right)$. Substituting this to (4.10) and using Lemma 4.1 completes the proof.

Lemma 4.2. Let $\rho \in S_{\nu}$ be such that $\Delta_{k}(\rho) \neq 0$. Then any solution $y(x)$ of $(1.1)$ satisfying the conditions:

$$
y(x)=O\left((\rho x)^{\mu_{k}}\right), x \rightarrow 0, y(x)=\exp \left(\rho R_{k} x\right)\left(\mathfrak{f}_{k}+o(1)\right), x \rightarrow \infty
$$

necessarily coincides with $\psi_{k}(x, \rho)$.

Proof. We consider the following tensors: $Y_{-}(x):=\left(y(x)-\psi_{k}(x, \rho)\right) \wedge T_{k}(x, \rho)$ and $Y_{+}(x):=F_{k-1}(x, \rho) \wedge\left(y(x)-\psi_{k}(x, \rho)\right)$. Then $Y_{-}$satisfies the equation:

$$
Y_{-}^{\prime}=Q_{0}^{(n-k+2)}(x, \rho) Y_{-}+q^{(n-k+2)}(x) Y_{-}
$$

and the estimate:

$$
\left\|Y_{-}(x)\right\| \leq C\left|(\rho x)^{\overleftarrow{\mu_{k}}+\mu_{k}}\right|,|\rho x| \leq 1
$$

Proceeding as in the proof of Lemma 4.1 we conclude that $Y_{-}(x)$ satisfies the following homogeneous Volterra equation:

$$
Y_{-}(x)=\int_{0}^{x} G_{n-k+2}(x, t, \rho)\left(q^{(n-k+2)}(t) Y_{-}(t)\right) d t
$$

that yields $Y_{-}=0$.

Similarly for $Y_{+}$we have:

$$
Y_{+}^{\prime}=Q_{0}^{(k)}(x, \rho) Y_{+}+q^{(k)}(x) Y_{+}, Y_{+}(x)=o\left(\exp \left(\rho x \vec{R}_{k}\right)\right), x \rightarrow \infty .
$$

This yields:

$$
Y_{+}(x)=\int_{x_{0}}^{x} G_{k}(x, t, \rho)\left(q^{(k)}(t) Y_{+}(t)\right) d t+\sum_{\alpha \in \mathcal{A}_{k}} A_{\alpha} \Psi_{\alpha}^{0}(x, \rho),
$$




$$
\left|A_{\alpha}\right|=\left\|Y_{+}\left(x_{0}\right) \wedge \Psi_{\alpha^{\prime}}^{0}\left(x_{0}, \rho\right)\right\|
$$

The asymptotics of $Y_{+}$implies $A_{\alpha}=o(1)$ as $x_{0} \rightarrow \infty$ for any $\alpha \in \mathcal{A}_{k}$. Thus, taking the limit as $x_{0} \rightarrow \infty$ we arrive at the following homogeneous Volterra equation:

$$
Y_{+}(x)=-\int_{x}^{\infty} G_{k}(x, t, \rho)\left(q^{(k)}(t) Y_{+}(t)\right) d t
$$

Repeating again the arguments used in the proof of Theorem 4.1 we obtain $Y_{+}=0$.

Thus we have $F_{k-1} \wedge\left(y-\psi_{k}\right)=0$ and $\left(y-\psi_{k}\right) \wedge T_{k}=0$. Since $F_{k-1} \wedge T_{k} \neq 0$, this means $y-\psi_{k}=0$.

Lemma 4.2 shows that for $\rho \in S_{\nu}$ such that $\Delta_{k}(\rho) \neq 0$ the $k$-th Weyl type solution exists, is unique and coincides with $\psi_{k}(x, \rho)$.

The rest of this section is devoted to investigation of the properties of Weyl-type solutions as the functions of spectral parameter $\rho$.

Theorem 4.3. For $\rho \rightarrow \infty, \rho \in \bar{S}_{\nu}$ the functions $\Delta_{k}(\rho)$ admit the asymptotics:

$$
\Delta_{k}(\rho)=\Delta_{k}^{0}+O\left(\rho^{-\varepsilon}\right), \varepsilon \in(0,1) .
$$

For any fixed $x$ and $\rho \rightarrow \infty, \rho \in \bar{S}_{\nu}$ the following asymptotics hold:

$$
\psi_{k}(x, \rho)=\sum_{j=1}^{k} \gamma_{j k}^{0} \exp \left(\rho x R_{j}\right) \mathfrak{f}_{j}+O\left(\rho^{-\varepsilon} \exp \left(\rho x R_{k}\right)\right)
$$

with constants $\gamma_{j k}^{0}, \gamma_{k k}^{0}=1$ that do not depend on $q(\cdot)$.

Proof. Using the representation $\Delta_{k}(\rho)=\left|F_{k-1}(x, \rho) \wedge T_{k}(x, \rho)\right|$, Theorem 3.1 and Corollary 3.1 we obtain the desired asymptotics for $\Delta_{k}(\rho)$ via direct calculation.

Now we use the induction in $k$ to prove the asymptotics for $\psi_{k}(x, \rho)$. For $k=1$ the asymtotics follows directly from Corollary 3.1. Now we suppose that for some $k>1$ all the functions $\psi_{1}(x, \rho), \ldots, \psi_{k-1}(x, \rho)$ are already shown to have the asymptotics specified in the theorem. The induction step consists in obtaining the asymptotics for $\psi_{k}(x, \rho)$.

First we notice that for any fixed $x$ and $\rho \rightarrow \infty, \rho \in \bar{S}_{\nu}$ we have:

$$
\left|\psi_{1} \wedge \ldots \wedge \psi_{k-1} \wedge E_{k} \wedge \ldots \wedge E_{n}\right|=\left|E_{1} \wedge \ldots \wedge E_{n}+O\left(\rho^{-\varepsilon}\right)\right|=\operatorname{det} \Pi_{\nu}+O\left(\rho^{-\varepsilon}\right) .
$$

Thus for any sufficiently large $\rho$ the vectors $\psi_{1}, \ldots, \psi_{k-1}, E_{k}, \ldots, E_{n}$ form a basis in $\mathbb{C}^{n}$ and we can use the expansion:

$$
\psi_{k}(x, \rho)=\sum_{j<k} \beta_{j k}(x, \rho) \psi_{j}(x, \rho)+\sum_{j \geq k} \beta_{j k}(x, \rho) E_{j}(x, \rho) .
$$

Then the relation $F_{k-1} \wedge \psi_{k}=F_{k}$ becomes:

$$
\sum_{j \geq k} \beta_{j k} F_{k-1} \wedge E_{j}=F_{k}
$$

and we find:

$$
\begin{gathered}
\beta_{k k}\left|F_{k-1} \wedge E_{k} \wedge \ldots \wedge E_{n}\right|=\left|F_{k} \wedge E_{k+1} \wedge \ldots \wedge E_{n}\right| \\
\left|\beta_{j k}\right| \cdot\left\|F_{k-1} \wedge E_{k} \wedge \ldots \wedge E_{n}\right\|=\left\|F_{k} \wedge E_{k} \wedge \ldots \wedge E_{j-1} \wedge E_{j+1} \wedge \ldots E_{n}\right\|, j>k .
\end{gathered}
$$


Using Corollary 3.1 and taking into account that $\left|F_{k-1}^{0} \wedge E_{k} \wedge \ldots \wedge E_{n}\right|=\left|F_{k}^{0} \wedge E_{k+1} \wedge \ldots \wedge E_{n}\right|$, $F_{k}^{0} \wedge E_{k}=0$ we calculate:

$$
\begin{aligned}
& \beta_{k k}(x, \rho)=1+O\left(\rho^{-1}\right), \beta_{j k}(x, \rho)=O\left(\rho^{-1} \exp \left(\rho x\left(R_{k}-R_{j}\right)\right)\right), \\
& \psi_{k}^{1}(x, \rho):=\sum_{j \geq k} \beta_{j k}(x, \rho) E_{j}(x, \rho)=E_{k}(x, \rho)+O\left(\rho^{-1} \exp \left(\rho x R_{k}\right)\right) .
\end{aligned}
$$

We rewrite (4.11) as follows:

$$
\psi_{k}(x, \rho)=\sum_{j<k} \beta_{j k}(x, \rho) \psi_{j}(x, \rho)+\psi_{k}^{1}(x, \rho) .
$$

Then the relation $\psi_{k} \wedge T_{k}=0$ becomes:

$$
\sum_{j<k} \beta_{j k} \psi_{j} \wedge T_{k}=-\psi_{k}^{1} \wedge T_{k}
$$

and we calculate:

$$
(-1)^{\chi_{j k}} \beta_{j k} \Delta_{k}=\left|\tilde{F}_{k-1, j} \wedge \psi_{k}^{1} \wedge T_{k}\right|, \chi_{j k} \in\{0,1\}
$$

Here we denote $($ for $j \leq m) \tilde{F}_{m, j}:=\psi_{1} \wedge \ldots \psi_{j-1} \wedge \psi_{j+1} \wedge \ldots \wedge \psi_{m}$ and take into account that $\left|F_{k-1} \wedge T_{k}\right|=\Delta_{k}$. Now we notice that the induction assumption implies, in particular, $\psi_{j}=\Psi_{j}^{0}+O\left(\rho^{-\varepsilon} \exp \left(\rho x R_{j}\right)\right), j<k$ (since coefficients in the asymptotics do not depend on $q(\cdot)$ and should be the same for the unperturbed equation). Therefore we have:

$$
\begin{gathered}
\tilde{F}_{k-1, j}(x, \rho)=\tilde{F}_{k-1, j}^{0}(x, \rho)+O\left(\rho^{-\varepsilon} \exp \left(\rho x\left(\vec{R}_{k-1}-R_{j}\right)\right)\right), \\
\tilde{F}_{k-1, j} \wedge \psi_{k}^{1}=\tilde{F}_{k-1, j}^{0} \wedge E_{k}+O\left(\rho^{-\varepsilon} \exp \left(\rho x\left(\vec{R}_{k}-R_{j}\right)\right)\right),
\end{gathered}
$$

where $\tilde{F}_{m, j}^{0}:=\Psi_{1}^{0} \wedge \ldots \Psi_{j-1}^{0} \wedge \Psi_{j+1}^{0} \wedge \ldots \wedge \Psi_{m}^{0}$. Thus, we can use Corollary 3.1 and Theorem 3.1 to obtain from (4.14) the following asymptotics:

$$
(-1)^{\chi j k} \beta_{j k} \Delta_{k}=\left|\tilde{F}_{k-1, j}^{0} \wedge E_{k} \wedge T_{k}^{0}\right|+O\left(\rho^{-\varepsilon} \exp \left(\rho x\left(R_{k}-R_{j}\right)\right)\right) .
$$

We notice again that the Wroskian $\left|\tilde{F}_{k-1, j}^{0} \wedge E_{k} \wedge T_{k}^{0}\right|$ of the solutions of (2.1) does not depend on $x, \rho$. Then from (4.15) it follows that:

$$
\beta_{j k}(x, \rho)=\beta_{j k}^{0}+O\left(\rho^{-\varepsilon} \exp \left(\rho x\left(R_{k}-R_{j}\right)\right)\right),
$$

where $\beta_{j k}^{0}$ obviously do not depend on $q(\cdot)$. Now we return to $(4.12),(4.13)$ and use again the induction assumption. This completes the proof.

Since the fundamental tensors $T_{k}(x, \rho), F_{k}(x, \rho) k=\overline{1, n}$ are analytical function of $\rho \in S_{\nu}$ and continuous in $\bar{S}_{\nu} \backslash\{0\}$, we can use our construction of the Weyl-type solutions to conclude that $\psi_{k}(x, \cdot)$ is holomorphic in $S_{\nu} \backslash Z_{\nu k}$ and continuous in $\bar{S}_{\nu} \backslash\left(Z_{\nu k} \cup\{0\}\right)$, where $Z_{\nu k}$ is a set of zeros of $\Delta_{k}(\cdot)$ in $\bar{S}_{\nu} \backslash\{0\}$.

The observations of Lemma 3.4 above allow us to evaluate a behavior of $\psi_{k}(x, \rho)$ for $\rho \rightarrow 0$. First we notice that $\vec{\mu}_{k-1}+\overleftarrow{\mu}_{k}=\sum_{j=1}^{n} \mu_{j}=0$ and therefore $\Delta_{k}(\rho)=F_{k-1}(x, \rho) \wedge T_{k}(x, \rho)=$ $\tilde{F}_{k-1}(x, \rho) \wedge \tilde{T}_{k}(x, \rho)$ are continuous w.r.t. $\rho \in \bar{S}_{\nu}$. 
Theorem 4.4. If for all $k=\overline{1, n} \Delta_{k}(0) \neq 0$ then all the functions $\rho^{-\mu_{k}} \psi_{k}(x, \rho)$ admit the continuous w.r.t. $\rho$ extensions to $\bar{S}_{\nu} \cap\{|\rho| \leq \delta\}$ for some positive $\delta$.

Proof. Our consideration uses induction in $k$. Suppose that $\tilde{\psi}_{j}(x, \rho):=\rho^{-\mu_{j}} \psi_{j}(x, \rho)$, $j=\overline{1, k-1}$ are already shown to be continuous in $\rho \in \bar{S}_{\nu} \cap\{|\rho| \leq \delta\}$ for some $\delta>0$. Then the induction step consists in proving that $\tilde{\psi}_{k}(x, \rho)$ is also continuous in $\rho \in \bar{S}_{\nu} \cap\{|\rho| \leq \delta\}$.

Further we assume that $x>0$ is arbitrary fixed and omit it in all the argument's lists.

At this point we have:

$$
\tilde{F}_{k}(\rho)=\tilde{\psi}_{1}(\rho) \wedge \ldots \wedge \tilde{\psi}_{k}(\rho), \rho \in \bar{S}_{\nu} \cap\{|\rho| \leq \delta\} \backslash\{0\},
$$

while the induction assumption yields:

$$
\tilde{F}_{k-1}(\rho)=\tilde{\psi}_{1}(\rho) \wedge \ldots \wedge \tilde{\psi}_{k-1}(\rho), \rho \in \bar{S}_{\nu} \cap\{|\rho| \leq \delta\} .
$$

From (4.16) it follows that

$$
\tilde{\psi}_{j}(\rho) \wedge \tilde{F}_{k}(\rho)=0, j=\overline{1, k-1}, \rho \in \bar{S}_{\nu} \cap\{|\rho| \leq \delta\} \backslash\{0\}
$$

But the left-hand side of (4.17) is continuous in $\rho \in \bar{S}_{\nu} \cap\{|\rho| \leq \delta\}$ therefore the relation is true for $\rho=0$ as well. By virtue of Lemma $7.1[9$ there exists a unique vector-function $f(\rho)$ that is orthogonal to all $\tilde{\psi}_{j}(\rho), j=\overline{1, k-1}$ and such that $\tilde{F}_{k-1} \wedge f=\tilde{F}_{k}$ for $\rho \in \bar{S}_{\nu} \cap\{|\rho| \leq \delta\}$.

Since $\Delta_{k-1}(0) \neq 0$, it is possible to choose some fixed vectors $\mathfrak{u}_{k}, \ldots, \mathfrak{u}_{n}$ such that $\tilde{\psi}_{1}(\rho) \wedge$ $\ldots \wedge \tilde{\psi}_{k-1}(\rho) \wedge \mathfrak{u}_{k} \wedge \ldots \wedge \mathfrak{u}_{n} \neq 0$ for all sufficiently small $\rho \in \bar{S}_{\nu}$. Therefore for all sufficiently small $\rho \in \bar{S}_{\nu} f(\rho)$ admits the following representation:

$$
f(\rho)=\sum_{j<k} \gamma_{j}(\rho) \tilde{\psi}_{j}(\rho)+\sum_{j \geq k} \gamma_{j}(\rho) \mathfrak{u}_{j} .
$$

Relations $\left(f, \tilde{\psi}_{j}\right)=0, j=\overline{1, k-1}, \tilde{F}_{k-1} \wedge f=\tilde{F}_{k}$ are equivalent to some system of linear equations with respect to $\gamma_{j}, j=\overline{1, n}$ with the coefficients, which are continuous w.r.t. $\rho \in \bar{S}_{\nu}$. Condition $\tilde{\psi}_{j} \wedge \tilde{F}_{k}=0, j=\overline{1, k-1}$ allow us to convert this system into some $(k-1) \times(k-1)$ linear system, which is non-degenerate for all sufficiently small $\rho \in \bar{S}_{\nu}$. Thus we can conclude that $f(\rho)$ is continuous in $\rho \in \bar{S}_{\nu} \cap\{|\rho| \leq \delta\}$ with some $\delta>0$. Since for $\rho \in \bar{S}_{\nu} \backslash\{0\}$ we have $\tilde{F}_{k-1} \wedge\left(\tilde{\psi}_{k}-f\right)=0$, we find that $\tilde{\psi}_{k}$ admits the representation:

$$
\tilde{\psi}_{k}(\rho)=f(\rho)-\sum_{j<k} \beta_{j}(\rho) \tilde{\psi}_{j}(\rho), \rho \in \bar{S}_{\nu} \cap\{|\rho| \leq \delta\} \backslash\{0\} .
$$

Then the relation $\tilde{\psi}_{k} \wedge \tilde{T}_{k}=0$ becomes:

$$
\sum_{j<k} \beta_{j}(\rho) \tilde{\psi}_{j}(\rho) \wedge \tilde{T}_{k}(\rho)=f(\rho) \wedge \tilde{T}_{k}(\rho),
$$

that yields

$$
\begin{gathered}
\beta_{j}(\rho) \Delta_{k}(\rho)= \\
(-1)^{\chi_{j}}\left|f(\rho) \wedge \tilde{\psi}_{1}(\rho) \wedge \ldots \wedge \tilde{\psi}_{j-1}(\rho) \wedge \tilde{\psi}_{j+1}(\rho) \ldots \wedge \tilde{\psi}_{k-1}(\rho) \wedge \tilde{T}_{k}(\rho)\right|, \chi_{j} \in\{0,1\} .
\end{gathered}
$$

By virtue of the condition $\Delta_{k}(0) \neq 0$ and of the induction assumption this relation defines the coefficients $\beta_{j}(\rho)$ as some continuous function of $\rho \in \bar{S}_{\nu} \cap\{|\rho| \leq \delta\}$. Thus the right-hand side in (4.18) is a continuous function of $\rho \in \bar{S}_{\nu} \cap\{|\rho| \leq \delta\}$ and the induction step is verified. 


\section{Inverse scattering problem. Uniqueness result.}

Here we consider the inverse scattering problem for system (1.1). For the sake of simplicity, we restrict our consideration with "solitonless" case. Specifically, we assume that the following condition is satisfied.

Condition $G_{0}$. For any sector $S_{\nu}, \nu=\overline{1, N}$ all the functions $\Delta_{k}(\rho), k=\overline{1, n}$ do not vanish for $\rho \in \bar{S}_{\nu}$.

For each sector $S_{\nu}, \nu=\overline{1, N}$ we consider the fundamental matrix

$$
\Psi(x, \rho):=\left(\psi_{1}(x, \rho), \ldots, \psi_{n}(x, \rho)\right),
$$

where $\psi_{k}(x, \rho)$ are the Weyl-type solutions of (1.1) constructed in the previous section. Let $\Sigma_{\nu}$ be a ray that separates the sectors $S_{\nu}$ and $S_{\nu+1}$ (we assume that the sectors $S_{1}, S_{2}, \ldots$ are enumerated in counterclockwise order and $S_{N+1}:=S_{1}$ ). Under Condition $G_{0}$ for any $\rho \in \Sigma_{\nu}$ there exist the boundary values $\Psi_{-}(x, \rho):=\lim _{\xi \rightarrow \rho, \xi \in S_{\nu}} \Psi(x, \xi)$ and $\Psi_{+}(x, \rho):=$ $\lim _{\xi \rightarrow \rho, \xi \in S_{\nu+1}} \Psi(x, \xi)$. We define for $\rho \in \Sigma_{\nu}$ the matrix $v(\rho):=\Psi_{-}^{-1}(x, \rho) \Psi_{+}(x, \rho)$. Following the general scheme of [9] we treat $v(\cdot)$ as scattering data and consider the following inverse scattering problem.

Problem IP0. Given $v(\rho), \rho \in \Sigma \backslash\{0\}, A, B$ recover $q(x), x \in(0, \infty)$.

In the sequel together with system (1.1) with the matrix coefficient $q(\cdot)$ we consider the system of the same form but with different (in general) coefficient $\tilde{q}(\cdot)$. We agree that if a symbol $\xi$ denotes some object related to system (1.1) with the coefficient $q(\cdot)$ then $\tilde{\xi}$ denotes an analogous object related to the system with the coefficient $\tilde{q}(\cdot)$.

Main result of this section is the following theorem that asserts that under conditions $R_{0}$ and $G_{0}$ specification of the matrix $v(\rho), \rho \in \Sigma \backslash\{0\}$ determines uniquely the coefficient $q(x)$, $x \in(0, \infty)$.

Theorem 5.1. Let the matrix $A, B$ be such that Condition $R_{0}$ is satisfied. Let $q(\cdot)$ and $\tilde{q}(\cdot)$ be such that Condition $G_{0}$ is satisfied. Then $\tilde{v}(\rho)=v(\rho)$ for all $\rho \in \Sigma \backslash\{0\}$ implies $\tilde{q}(x)=q(x)$ for a.e. $x \in(0, \infty)$.

Proof. Let us consider the following spectral mappings matrix[10]:

$$
P(x, \rho):=\Psi(x, \rho) \tilde{\Psi}^{-1}(x, \rho) .
$$

Under the conditions of theorem for each fixed $x \in(0, \infty) P(x, \cdot)$ is a sectionary-holomorphic function with jump matrices on $\Sigma_{\nu}, \nu=\overline{1, N}$ equal to:

$$
P_{-}^{-1}(x, \rho) P_{+}(x, \rho)=\tilde{\Psi}_{-}(x, \rho) v(\rho) \tilde{v}^{-1}(\rho) \tilde{\Psi}_{-}^{-1}(x, \rho) .
$$

If $\tilde{v}=v$ then we have $P_{+}(x, \rho)=P_{-}(x, \rho)$ for all $\rho \in \Sigma \backslash\{0\}$. Therefore $P(x, \cdot)$ is holomorphic in all $\rho$-plane with possible exception of $\rho=0$. Moreover, by virtue of Theorem 4.4 $P(x, \rho)=\left(\Psi(x, \rho) \rho^{-\mu}\right)\left(\tilde{\Psi}(x, \rho) \rho^{-\mu}\right)^{-1}\left(\right.$ where $\left.\mu:=\operatorname{diag}\left(\mu_{1}, \ldots, \mu_{n}\right)\right)$ is bounded as $\rho \rightarrow 0$. Furthermore, by virtue of Theorem 4.3 we have

$$
\Psi(x, \rho)=\left(\mathfrak{f} \Gamma^{0}(x, \rho)+O\left(\rho^{-\varepsilon}\right)\right) \exp (\rho x R),
$$

where $\mathfrak{f}:=\left(\mathfrak{f}_{1}, \ldots, \mathfrak{f}_{n}\right), R:=\operatorname{diag}\left(R_{1}, \ldots, R_{n}\right), \Gamma_{j k}^{0}(x, \rho)=\gamma_{j k}^{0} \exp \left(\rho x\left(R_{j}-R_{k}\right)\right)$ for $j \leq k$ and $\Gamma_{j k}^{0}(x, \rho)=0$ for $j>k$. Since for $\rho \in \bar{S}_{\nu}$ and $j \leq k$ we have $\operatorname{Re}\left(\rho\left(R_{j}-R_{k}\right)\right) \leq 0$ and $\gamma_{k k}^{0}=1$, we can estimate $\Gamma^{0}(x, \rho)=O(1)$ and $\left(\Gamma^{0}(x, \rho)\right)^{-1}=O(1)$. Therefore

$$
\Psi(x, \rho)=\mathfrak{f} \Gamma^{0}(x, \rho)\left(I+O\left(\rho^{-\varepsilon}\right)\right) \exp (\rho x R) .
$$


Since the matrix $\Gamma^{0}(x, \rho)$ does not depend on $q(\cdot)$, this yields $P(x, \rho)=I+O\left(\rho^{-\varepsilon}\right)$ as $\rho \rightarrow \infty$. Thus, we can conclude that $P(x, \rho) \equiv I, \tilde{\Psi}(x, \rho)=\Psi(x, \rho)$ and finally $\tilde{q}(x)=q(x)$ for a.e. $x$.

Acknowledgement. This work was supported by Grant 1.1436.2014K of the Russian Ministry of Education and Science and by Grants 15-01-04864 and 16-01-00015 of Russian Foundation for Basic Research.

\section{References}

[1] Brunnhuber R., Kostenko A. and Teschl G., Singular Weyl-Titchmarsh-Kodaira theory for one-dimensional Dirac operators, Monatshefte für Mathematik (2014), DOI: 10.1007/s00605-013-0563-5.

[2] Albeverio S., Hryniv R. and Mykytyuk Ya., Reconstruction of radial Dirac operators, J. Math. Phys. 48(2007), 043501, 14p.

[3] Albeverio S., Hryniv R. and Mykytyuk Ya., Reconstruction of radial Dirac and Schrödinger operators from two spectra, J. Math. Anal. Appl. 339(2008), 45-57.

[4] Serier F., Inverse spectral problem for singular Ablowitz-Kaup-Newell-Segur operators on $[0 ; 1]$, Inverse Problems 22(2006), 1457-1484.

[5] Gorbunov O.B., Shieh C.-T. and Yurko V.A., Dirac system with a singularity in an interior point., Applicable Analysis (2015), 17pp. DOI: 10.1080/00036811.2015.1091069.

[6] Beals R. and Coifman R.R., Scattering and inverse scattering for first order systems, Comm. Pure Appl. Math. 37(1984), 39-90.

[7] Zhou X., Direct and inverse scattering transforms with arbitrary spectral singularities, Comm. Pure Appl. Math. 42 (1989), 895-938.

[8] Yurko V.A., Inverse spectral problems for differential systems on a finite interval, Results in Math. 48, N. 3-4 (2006), 371-386.

[9] Beals R., Deift P. and Tomei C., Direct and inverse scattering on the line, American Mathematical Society, Providence, Rhod Island (1988).

[10] Yurko V.A., Method of Spectral Mappings in the Inverse Problem Theory. Inverse and Ill-Posed Problems Series, Utrecht: VSP (2002).

[11] Yurko V.A., On higher-order differential operators with a singular point, Inverse Problems. 9 (1993), 495-502.

[12] Fedoseev A. E. Inverse problems for differential equations on the half-line having a singularity in an interior point, Tamkang J. of Math. 42(2011), no. 3, 343-354.

Ignatyev, Mikhail

Department of Mathematics, Saratov State University, Astrakhanskaya 83, Saratov 410012, Russia, e-mail: mikkieram@gmail.com, ignatievmu@info.sgu.ru 